\title{
Nouvelles faunes de rongeurs (Mammalia, Rodentia) d'âge miocène moyen en Languedoc-Roussillon (sud de la France); biostratigraphie et corrélations
}

\author{
Jean-Pierre AGUILAR \\ Jacques MICHAUX \\ Université Montpellier 2, UMR-CNRS 5554, \\ Institut des Sciences de l'Évolution, place E. Bataillon, cc 064, \\ F-34095 Montpellier cedex 05 (France) \\ jean-pierre.aguilar@univ-montp2.fr \\ jacques-jules.michaux@univ-montp2.fr \\ Vincent LAZZARI \\ Université de Poitiers, UMR-CNRS 6046, IPHEP, \\ 40 avenue du Recteur Pineau, F-86022 Poitiers (France) \\ vincent.lazzari@univ-poitiers.fr
}

MOTS CLÉS

Mammalia,

Rodentia,

Miocène moyen, sud de la France,

corrélations,
Aguilar J.-P., Michaux J. \& Lazzari V. 2010. - Nouvelles faunes de rongeurs (Mammalia, Rodentia) d'âge miocène moyen en Languedoc-Roussillon (sud de la France); biostratigraphie et corrélations. Geodiversitas 32 (3): 501-513.

\section{RÉSUMÉ}

Les rongeurs recueillis dans les sites de Blanquatère 3 (Roussillon; remplissage de fissure karstique) et de Puisserguier (Languedoc; dépôt laguno-lacustre), d'âge miocène moyen, permettent de placer ces gisements entre celui, plus ancien, de Blanquatère 1 (Roussillon) et celui, plus récent, de La Grenatière (Languedoc). La présence du rongeur Cricetodon dans ces deux sites permet de discuter les corrélations admises jusqu'ici avec les gisements lyonnais classiques de VieuxCollonges et de La Grive: tous deux sont plus anciens que ceux de La Grive et Blanquatère 3 est légèrement plus ancien que Vieux-Collonges. Le niveau de Puisserguier est interstratifié dans une formation marine, que terminent des dépôts laguno-lacustres dans lesquels se situe le gisement de La Grenatière. Les données combinées relatives aux dépôts marins de l'Ouest biterrois et au sondage Tramontane 1 s'accordent pour attribuer ces formations à l'étage Serravallien, les termes les plus récents relevant de la biozone NN9 (sondage) et, sur le continent, de la biozone NN6, la formation laguno-lacustre qui affleure ne pouvant être plus récente que celle traversée par le sondage en mer. Compte tenu de ces informations et des âges numériques relevés dans la littérature (données magnétostratigraphiques ou radiométriques de l'étalonnage du Miocène) il est possible d'avancer un âge numérique pour ces nouveaux gisements: Blanquatère 3 aurait 15,5 Ma et Puisserguier $14 \mathrm{Ma}$. 


\begin{abstract}
New Middle Miocene rodent faunas from Languedoc-Roussillon (southern France); implication for biostratigraphic correlations.

The fossil mammal bearing localities Blanquatère 3 (Roussillon; filling of a fissure in a karst) and Puisserguier (Languedoc; brackish to lake deposit) have provided Middle Miocene rodents that first allow correlating these sites to Blanquatère 1 (Roussillon; older) and La Grenatière (Languedoc; younger). Second, the occurrence of the genus Cricetodon in these newly discovered localities similarly allow revisiting the correlations already proposed with the well-known localities from the Lyon area, Vieux-Collonges and La Grive. Blanquatère 3 and Puisserguier are older than La Grive, and Blanquatère 3 is slightly older than Vieux-Collonges. The fossil bearing levels of Puisserguier is interstratified in a mainly marine succession ending with brackish to lake deposits, which includes the level of La Grenatière. The onshore (Western Béziers area) and offshore (Tramontane 1 drilling) data on the marine Miocene agree referring these deposits to the Serravallian stage, the uppermost marine levels belonging to zone NN9 (drill) and NN6 (onshore), the uppermost brackish to lake deposits cannot be younger that the Miocene deposits encountered by the drill. According to the new collected data on one side, and the Miocene chronostratigraphy, it can be hypothesized that Blanquatère 3 is $c .15 .5 \mathrm{Myr}$ old and Puisserguier, $c .14 \mathrm{Myr}$.

\author{
KEY WORDS \\ Mammalia, \\ Rodentia, \\ correlations, \\ biostratigraphy.
}

Middle Miocene, southern France,
\end{abstract}

\section{INTRODUCTION}

S'il faut souligner l'efficacité reconnue de la biochronologie établie à partir des faunes de mammiferres, il faut également tenir compte de ses limitations. En particulier, l'absence de contrôle indépendant des corrélations (géométrie des dépôts, datations numériques à partir des radio-isotopes) affaiblit parfois la valeur des datations proposées. $S$ 'ajoutent à cela les difficultés dues aux sites fossiliferes eux-mêmes qui ne livrent souvent que des faunes pauvres en spécimens comme en espèces. La collecte de restes de mammiferes dans un gisement revient en effet à un échantillonnage plus ou moins imparfait de l'histoire d'une faune régionale. Le biais introduit est d'autant plus grand que le nombre des espèces déterminées est modeste et que l'on doit, pour la dater, corréler avec des faunes issues de régions éloignées. En effet, des facteurs environnementaux ont pu affecter la répartition ou l'abondance des espèces dont les populations illustrent une variation plus ou moins forte. Une telle situation explique une grande part des polémiques qui opposent les spécialistes. Néanmoins, malgré ces difficultés, des résultats intéressants sont aussi obtenus et c'est un nouvel exemple qui est rapporté dans le présent travail.

À ce jour, les nombreux sites à micromammiferes du Mio-Pliocène en Languedoc-Roussillon ont permis d'établir une biochronologie relativement fine (pouvoir de résolution estimée de l'ordre de $0,5 \mathrm{Ma}$ ) sur la base de quelques lignées de rongeurs qui se relaient les unes les autres dans cet intervalle de temps. L'histoire de la faune restituée révèle des changements de composition marqués en particulier par des migrations et des extinctions (Aguilar $e t$ al. 1999b; Lazzari \& Aguilar 2007; Lazzari et al. 2007). En même temps, une corrélation avec l'échelle stratigraphique marine est venue compléter cette biochronologie (Aguilar 1982; Aguilar \& Michaux 1987; Lazzari \& Aguilar 2007). Parmi les acquis relatifs à la composition des faunes, à l'échelle du sud de la France, les faunes de rongeurs du Miocène moyen en Languedoc-Roussillon se démarquaient jusqu'ici de celles des sites de la région lyonnaise par l'absence quasi totale de représentants du genre Cricetodon Lartet, 1851, l'unique exception étant la localité de 
Luc-sur-Orbieu (Aguilar \& Magné 1978; Aguilar 1980). La découverte de ce genre dans deux nouveaux sites s'avère donc particulièrement intéressante à exploiter dans la mesure où, une fois ces sites datés, la possibilité est donnée de revenir sur les corrélations entre chronologie marine et mammalienne.

\section{MATÉRIEL ET MÉTHODES}

Les mensurations des dents ont été effectuées sous une loupe binoculaire Wild, à l'aide d'une platine de mesures Leica, elles sont exprimées en millimètres. Le matériel est déposé dans les collections de l'Université Montpellier 2.

\section{LES GISEMENTS ET LEUR FAUNE}

LE GISEMENT DE BLANQUATÈRE 3 (BLQ 3)

Commune de Tautavel (Roussillon), à $15 \mathrm{~m} \mathrm{du}$ gisement de Blanquatère 1.

Ce site karstique a été découvert à l'occasion des prospections autour du gisement de Blanquatère 1 (Aguilar \& Lazzari 2006; Lazzari \& Aguilar 2007; Lazzari et al. 2007). La microfaune provient de trois blocs qui, récoltés en surface, présentent une composition sédimentaire identique, laquelle differe nettement de celle des autres remplissages de fissures proches. Un placage encore visible dans une fissure étroite entièrement vidée proche du lieu où les blocs ont été trouvés témoignerait du remplissage d'où sont issus les trois blocs analysés, placage qui n'a toutefois livré que quelques débris d'os et d'incisives de rongeurs. La faune est présentée ci-après.

Cricetodon cf. meini Freudenthal, 1963

(Fig. 1A)

Matériel et dimensions. $-1 \mathrm{~m} 1(-\times 1,87)$ et $1 \mathrm{~m} 2(2,44 \times 1,84)\left(\right.$ BLQ $3 \mathrm{n}^{\circ} 2$ et 3$)$.

\section{Discussion}

Cette m2, caractéristique du genre Cricetodon présente une morphologie et des dimensions comparables à celles de C. meini de Vieux-Collonges. Quant à la m1, cassée au niveau de l'antéroconide, elle présente une largeur qui entre également dans le domaine de variation de C. meini (Mein \& Freudenthal 1971b).

\section{Cricetodon cf. aureus}

Mein \& Freudenthal, 1971

(Fig. 1B)

MATÉRIEL ET DIMENSIONS. $-1 \mathrm{~m} 2(2,66 \times 2,00)$ (BLQ $3 n^{\circ} 4$ ).

\section{Discussion}

Cette $\mathrm{m} 2$ est différente de l'autre $\mathrm{m} 2$ présente dans le gisement par des dimensions supérieures, supérieures même à celles de $C$. meini de Vieux-Collonges. En revanche, ces dimensions entrent dans le domaine de variation de celles de $C$. aureus présent également à Vieux-Collonges (Mein \& Freudenthal 1971b).

\section{Megacricetodon tautavelensis \\ Lazzari \& Aguilar, 2007}

(Fig. 1L-N)

MATÉRIEL ET DIMENSIONS. $-1 \mathrm{~m} 1(1,23 \times 080)$; $3 \mathrm{~m} 2(1,02 \times 0,81 ; 1,02 \times-; 1,05 \times 0,92) ; 1 \mathrm{~m} 3$ $(0,92 \times 0,78) ; 2 \mathrm{M} 2(1,07 \times 0,97 ;-\times 0,90)($ BLQ 3 no 33 à 39).

\section{Discussion}

Ce Megacricetodon Fahlbusch, 1964 de petite taille, montre une morphologie et des dimensions qui sont comparables à celles de l'importante population de Megacricetodon tautavelensis de Blanquatère 1 (Lazzari \& Aguilar 2007).

\section{Megacricetodon "collongensis»-gersii}

(Fig. 1F-K; Tableau 1)

Matériel et Dimensions. - Dents isolées (BLQ 3 no 5 à 32). Dimensions: voir Tableau 1.

\section{Discussion}

Aguilar (1995) a proposé de désigner ainsi sous ce nom, des populations trouvées dans le Roussillon qui illustrent un stade évolutif intermédiaire entre 
TABleAu 1. - Mensurations (en mm) des molaires de Megacricetodon «collongensis »-gersii Aguilar, 1995 du gisement de Blanquatère 3 (sud de la France). N, nombre de dents dont la longueur/ largeur est mesurable.

\begin{tabular}{ccccccccc}
\hline & & \multicolumn{3}{c}{ Longueur } & & \multicolumn{3}{c}{ Largeur } \\
\cline { 3 - 5 } & $\mathbf{N}$ & min & moy & max & & min & moy & max \\
\hline m1 & $9 / 7$ & 1,36 & 1,45 & 1,63 & & 0,83 & 0,88 & $>0,95$ \\
m2 & 2 & 1,26 & & 1,33 & & 1,02 & & 1,11 \\
m3 & 3 & 1,14 & 1,20 & 1,24 & & 0,94 & 0,96 & 1,00 \\
M1 & $4 / 8$ & 1,51 & 1,59 & 1,74 & & 0,93 & 0,99 & 1,08 \\
M2 & 4 & 1,12 & 1,16 & 1,18 & & 0,96 & 1,01 & 1,05 \\
M3 & 2 & 0,95 & & 1,02 & & 1,04 & & 1,06 \\
\hline
\end{tabular}

TABLEAU 2. - Mensurations (en mm) des molaires de Democricetodon aff. mutilus Fahlbusch, 1964 du gisement de Blanquatère 3 (sud de la France). $\mathbf{N}$, nombre de dents dont la longueur/ largeur est mesurable.

\begin{tabular}{|c|c|c|c|c|c|c|c|}
\hline & \multirow[b]{2}{*}{$\mathbf{N}$} & \multicolumn{3}{|c|}{ Longueur } & \multicolumn{3}{|c|}{ Largeur } \\
\hline & & $\min$ & moy & $\overline{\max }$ & $\min$ & moy & $\overline{\max }$ \\
\hline$\overline{\mathrm{m} 1}$ & 1 & & 1,68 & & & 1,09 & \\
\hline $\mathrm{m} 2$ & 1 & & 1,42 & & & 1,16 & \\
\hline m3 & 1 & & 1,17 & & & 0,98 & \\
\hline M1 & 4 & 1,84 & 1,90 & 2,01 & 1,25 & 1,28 & 1,32 \\
\hline M2 & $6 / 7$ & 1,33 & 1,40 & 1,52 & 1,22 & 1,26 & 1,35 \\
\hline M3 & 1 & & 1,13 & & & 1,17 & \\
\hline
\end{tabular}

celui plus primitif $M$. «collongensis» (Mein, 1958) de Port-la-Nouvelle et celui plus évolué $M$. gersii de Ste Catherine 1 et de Luc-sur-Orbieu. Il est apparu difficile de définir une espèce nouvelle car dans cette lignée $M$. «collongensis»-M. roussillonensis, la variabilité morphologique est importante et la différence principale s'observe à la moyenne des dimensions lorsque les populations sont abondantes (Aguilar 1995). Les quelques spécimens dentaires de Blanquatère 3 ne peuvent être différenciés de ceux de Blanquatère 1 (Lazzari \& Aguilar 2007) ni par la morphologie, ni par les dimensions.

Megacricetodon cf. aunayi Lazzari \& Aguilar, 2007 (Fig. 1D, E)

MatéRIel eT DIMENSIONS. - Dents isolées: $4 \mathrm{~m} 1$ $(1,91 \times 1,17 ;>1,76 \times 1,15 ;>1,68 \times 1,15 ;-\times 1,09)$; $1 \mathrm{~m} 2(1,44 \times 1,22) ; 1 \mathrm{~m} 3(1,30 \times 1,01) ; 1 \mathrm{M} 1(2,06$ $\times 1,29) ; 4$ M2 $(1,41 \times<1,33 ; 1,33 \times 1,24 ; 1,28 \times$ $1,20 ; 1,34 \times 1,30)$ (BLQ 3 no 40 à 50$)$.

\section{Discussion}

Par les caractères morphologiques et les dimensions, ces molaires sont comparables à celles du Megacricetodon de grande taille $M$. aunayi de Blanquatère 1 (Lazzari \& Aguilar 2007). Le matériel est incomplet et insuffisant pour que nous puissions l'attribuer sans conteste à $M$. aunayi.

\section{Pseudofahlbuschia aff. jordensis \\ Freudenthal \& Daams, 1988}

(Fig. 1T, U)

Matériel ET Dimensions. - Dents isolées: $3 \mathrm{~m} 2$ $(1,62 \times 1,23 ; 1,54 \times 1,27 ; 1,66 \times 1,33) ; 1 \mathrm{M} 1$ $(>1,84 \times>1,30)$ (BLQ 3 no 69 à 72).

\section{Discussion}

Ces quelques molaires sont comparables à celles de la petite population de Blanquatère 1 (Aguilar et al. 2010). Cette dernière présente des caractères qui la différencient à la fois de $P$. catalaunica Aguilar, 1999 des gisements de Baixas 200 et 201 (Aguilar 1999) et de P. jordensis du gisement ibérique de Villafeliche 4A (Freudenthal \& Daams 1988).

Democricetodon aff. mutilus Fahlbusch, 1964 (Fig. 1O-Q; Tableau 2)

Matériel ET Dimensions. - Dents isolées (BLQ 3 no 51 à 65). Dimensions: voir Tableau 2.

\section{Description}

Molaires inférieures. Le mésolophide est de longueur moyenne sur la m1 et court sur la m2.

Molaires supérieures. Le mésolophe est bien développé sur M1 et M2 sans toutefois atteindre le bord labial. Le protolophule est double sur les M2, cette double connexion est présente sur 2 M1, sur les deux autres le protolophule est postérieur. Le métalophule est postérieur sur les $4 \mathrm{M} 1$, alors que sur les M2 il est en majorité proverse à transverse, il n'est rétroverse que sur 2 dents. Toutes les M2 ont un antérolophe lingual plus ou moins développé. 
A

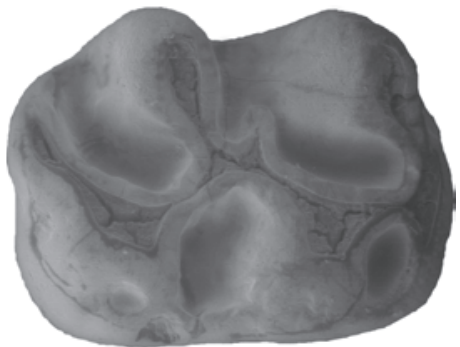

C

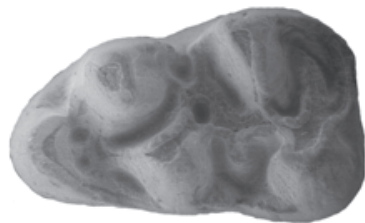

$\mathrm{F}$

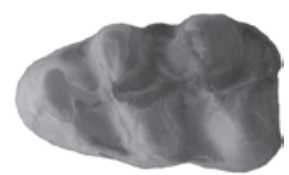

$J$

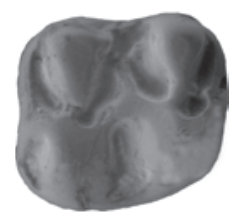

$\mathrm{O}$

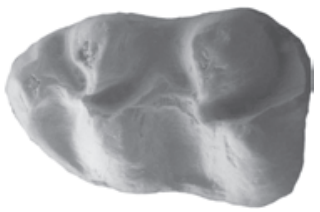

R

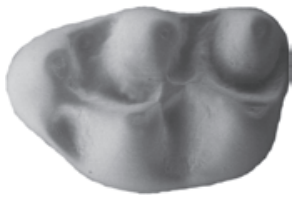

G

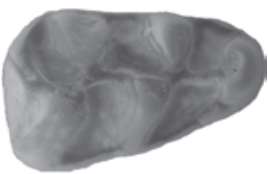

$\mathrm{K}$

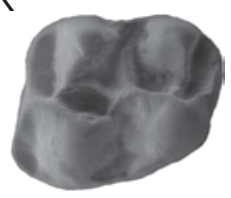

D

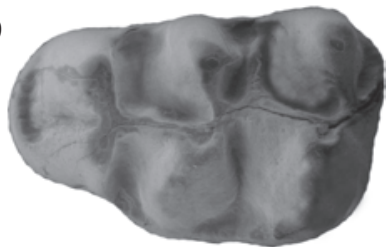

$\mathrm{H}$

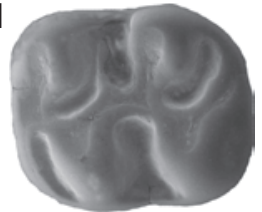

L

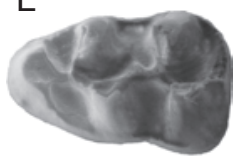

P

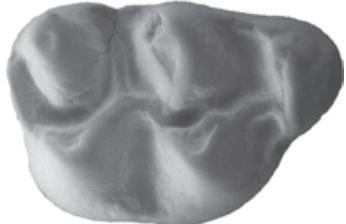

S

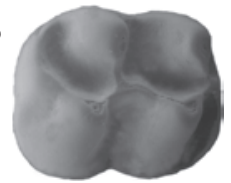

Q

$\mathrm{T}$

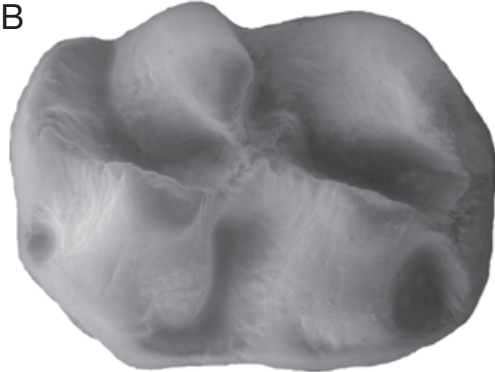

E

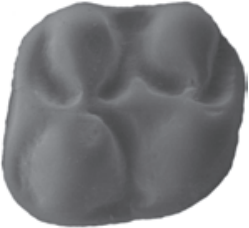

I
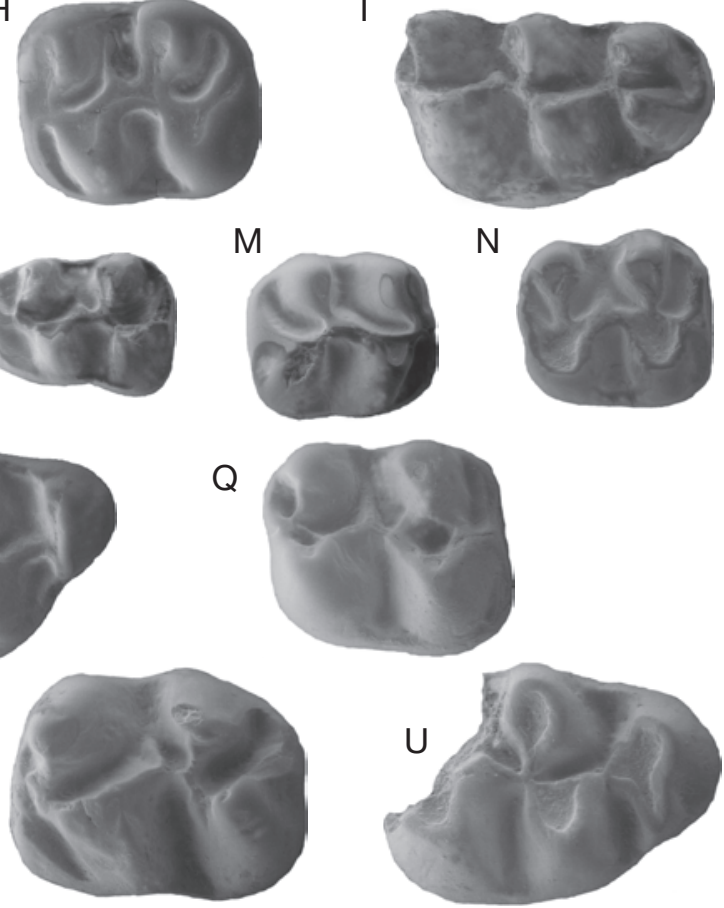

$\mathrm{N}$
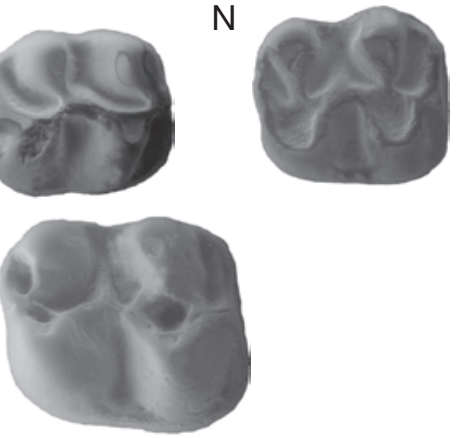

$\mathrm{M}$
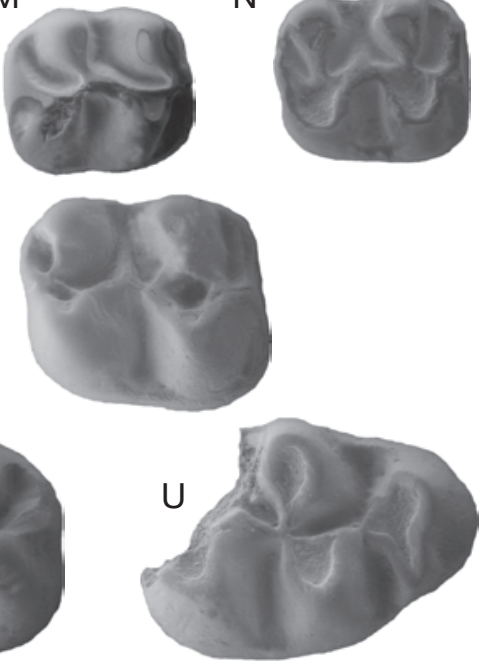

FIG. 1 - Molaires des rongeurs de Blanquatère 3 (mensurations en mm) : A, Cricetodon cf. meini Freudenthal, $1963, \mathrm{~m} 2$ dex., $2,44 \times$ 1,44 (BLQ $3 \mathrm{n}^{\circ}$ 3); B, Cricetodon cf. aureus Mein \& Freudenthal, 1971, m2 dex., 2,66 × 2,00 (BLQ $3 \mathrm{n}^{\circ}$ 4); C, Eumyarion cf. bifidus (Fahlbusch, 1964), m1 sen., 1,95 × 1,20 (BLQ 3 n 1); D, E, Megacricetodon cf. aunayi Lazzari \& Aguilar, 2007 ; D, M1 sen., 2,06 $\times$ 1,29 (BLQ $3 \mathrm{n}^{\circ}$ 46); E, M2 sen., 1,34 × 1,30 (BLQ $3 \mathrm{n}^{\circ}$ 50); F-K, Megacricetodon «collongensis »-gersii; $\mathbf{F}, \mathrm{m} 1$ sen., 1,46 $\times$ 0,88 (BLQ

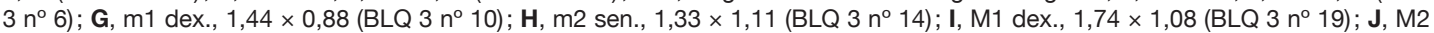
sen., 1,16 × 1,05 (BLQ $3 \mathrm{n}^{\circ}$ 28); K, M2 dex., 1,16 × 0,96 (BLQ $3 \mathrm{n}^{\circ}$ 30); L-N, Megacricetodon tautavelensis Lazzari \& Aguilar, 2007; L, $\mathrm{m} 1$ sen., 1,23 × 0,80 (BLQ $3 \mathrm{n}^{\circ}$ 33); M, m2 dex., 1,05 × 0,92 (BLQ $3 \mathrm{n}^{\circ}$ 36); N, M2 dex., 1,07 × 0,97 (BLQ $3 \mathrm{n}^{\circ} 38$ ); O-Q, Democricetodon aff. mutilus; $\mathbf{O}$, m1 sen., 1,68 × 1,09 (BLQ $3 n^{\circ}$ 51); P, M1 dex., 1,84 × 1,25 (BLQ $3 n^{\circ}$ 55); Q, M2 dex., 1,37 × 1,22 (BLQ $3 n^{\circ}$ 62); R, S, Democricetodon cf. gracilis Fahlbusch, 1964; R, M1 sen., 1,55 × 1,05 (BLQ $3 n^{\circ}$ 66); S, m2 dex., 1,15 × 0,97 (BLQ $3 n^{\circ} 67$ ); T, U, Pseudofahlbuschia aff. jordensis Freudenthal \& Daams, 1988; T, m2 sen., 1,62 × 1,23 (BLQ $3 \mathrm{n}^{\circ}$ 69); U, M1 dex. fragmentée, $>1,84 \times>1,30$ (BLQ $3 \mathrm{n}^{\circ}$ 72). Échelle: $1 \mathrm{~mm}$. 


\section{Discussion}

Ces molaires sont comparables à celles de Blanquatère 1 (Aguilar et al. 2010) ainsi qu'à celles des populations importantes recueillies dans les différents sites du Miocène moyen du Roussillon (Aguilar et al. 1999b) et qui doivent appartenir à une nouvelle espèce.

Democricetodon cf. gracilis Fahlbusch, 1964 (Fig. 1R, S)

MatéRiel et dimensions. - Dents isolées : $2 \mathrm{~m} 2$ $(1,15 \times 0,97 ;-\times 0,98) ; 1$ M1 $(1,55 \times 1,05)$ (BLQ 3 $\mathrm{n}^{\circ} 66$ à 68$)$.

\section{Discussion}

Ce Democricetodon Fahlbusch, 1964 de petite taille présente les caractères morphologiques que l'on observe dans les populations allemandes (Fahlbusch 1964) ainsi que dans celles du Roussillon (Aguilar et al. 1999, 2010).

Ces deux espèces de Democricetodon sont présentes à Blanquatère 1 ainsi que dans plusieurs sites du Miocène moyen en Languedoc-Roussillon (Aguilar et al. 1999, 2010).

\section{Eumyarion cf. bifidus (Fahlbusch, 1964)}

(Fig. 1C)

MATÉRIEl ET Dimensions. - $1 \mathrm{~m} 1(1,95 \times 1,20)$ (BLQ $3 n^{\circ} 1$ ).

\section{Discussion}

Cette espèce est représentée à Blanquatère 1 par quelques dents (Aguilar et al. 2010) et montre une morphologie et des dimensions comparables à celles des populations bien décrites dans les sites du Miocène moyen d'Allemagne (Wu 1982).

\section{Heteroxerus rubricati}

Crusafont, de Villalta \& Truyols, 1955

MATÉRIEL ET DIMENSIONS. - 1 p4 $(1,42 \times 1,27)$; $1 \mathrm{~m} 1(1,51 \times 1,38) ; 1 \mathrm{M} 3(1,63 \times 1,75)(\mathrm{BLQ} 3$ no 73 à 75$)$.

\section{Pseudodryomys ibericus De Bruijn, 1966}

MATÉRIEL ET DiMENSIONS. $-1 \mathrm{~m} 1(1,128 \times 1,18)$; $1 \mathrm{~m} 2(1,34 \times 1,32) ; 1 \mathrm{M} 1(1,18 \times>1,41)$ (BLQ 3 no 85 à 87$)$.

\section{Pseudodryomys cf. simplicidens}

De Bruijn, 1966

MatéRIEL ET Dimensions. $-1 \mathrm{~m} 1(1,24 \times 1,09)$; 1 M2 $(1,01 \times 1,23)($ BLQ 3 no 88 et 89$)$.

\section{Microdyromys aff. koenigswaldi}

De Bruijn, 1966

MatéRIel eT Dimensions. $-2 \mathrm{~m} 1(1,03 \times 0,99$; $0,97 \times 0,88) ; 1 \mathrm{~m} 2(1,02 \times 1,00) ; 1 \mathrm{M} 2(1,00 \times$ $1,20)$ (BLQ 3 no 76 à 79).

\section{Discussion}

Le sciuridé Heteroxerus rubricati et les trois espèces de gliridés sont bien représentées dans le gisement de Blanquatère 1 (Aguilar \& Lazzari 2006; Aguilar et al. 2010) et couramment citées dans les faunes du début du Miocène moyen (Aguilar et al. 1999b; Aguilar 2002).

Miodyromys cf. aegercii Baudelot, 1972

(Fig. 2A-C)

MatéRIel et Dimensions. - $1 \mathrm{~m} 1(1,29 \times 1,26)$; $2 \mathrm{M} 1(1,34 \times 1,50 ; 1,36 \times 1,52) ; 2 \mathrm{M} 2(1,30 \times 1,49$; $1,31 \times 1,64)($ BLQ 3 no 80 à 84).

\section{Description}

Molaires inférieures. Sur la m1, le centrolophide long dépasse le milieu de la couronne; il y a deux bourgeons d'émail en position linguale entre l'antérolophide et le métalophide et entre le métalophide et le centrolophide; la crête accessoire postérieure est bien développée.

Molaires supérieures. Aux molaires supérieures deux centrolophes sont présents, le centrolophe antérieur est plus développé que le postérieur; la crête accessoire antérieure est bien développée. 
A

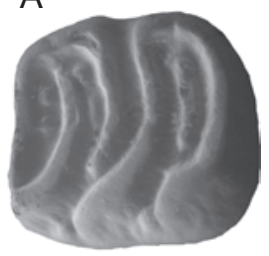

B

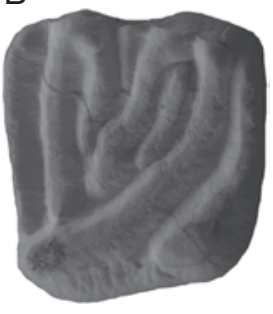

C

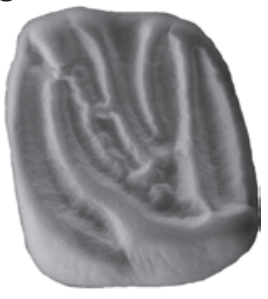

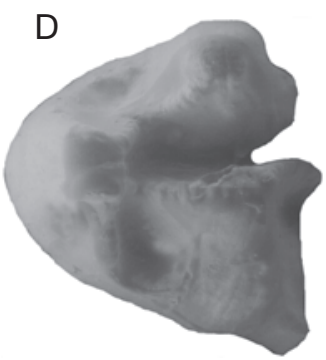

E

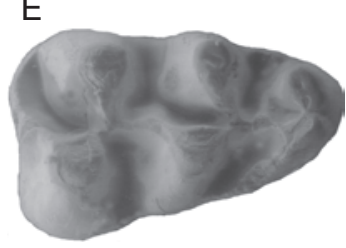

$\mathrm{H}$
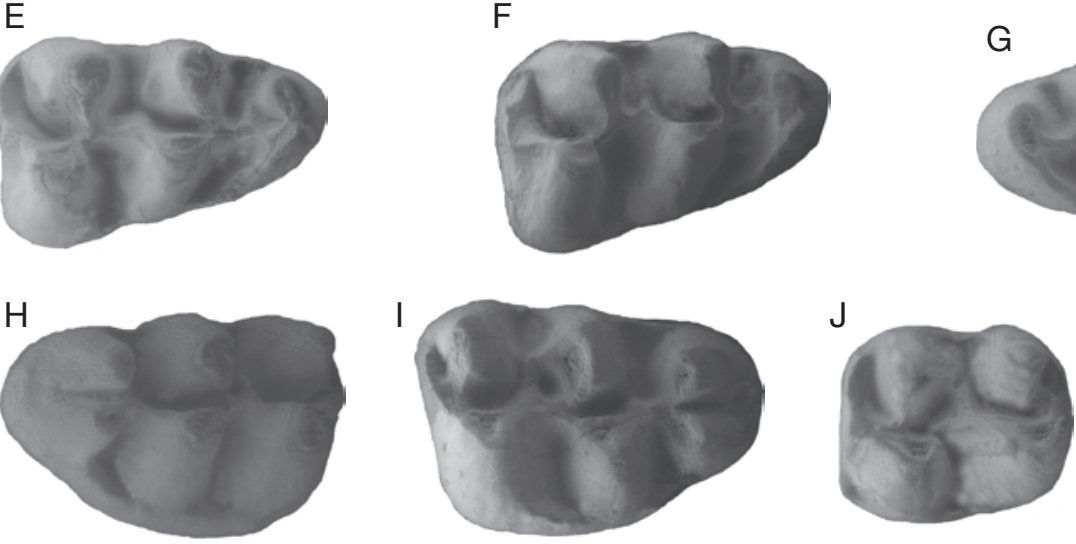

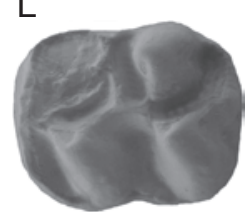

M

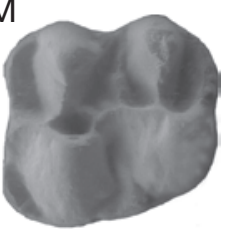

N

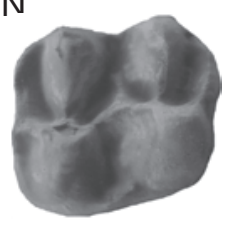

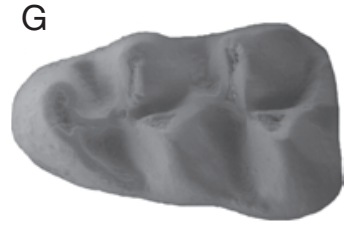

K

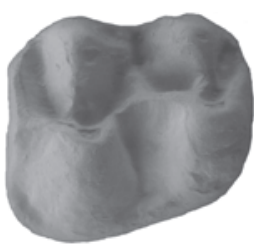

$\mathrm{O}$

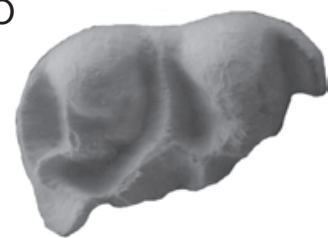

FIG. 2 - A-C, molaires des rongeurs de Blanquatère 3 (mensurations en mm); A-C, Miodyromys cf. aegercii Baudelot, 1972; A, $\mathrm{m} 1$ sen., 1,29 × 1,26 (BLQ 3 n 80); B, M1 dex., 1,36 × 1,52 (BLQ $3 \mathrm{n}^{\circ}$ 82); C, M2 sen., 1,30 × 1,49 (BLQ 3 n 83); D-O, molaires des rongeurs de Puisserguier (mensurations en mm); D, Cricetodon cf. albanensis Mein \& Freudenthal, 1971, m1 sen. fragmentée; E-K, Megacricetodon aff. fournasi Aguilar, 1995; E, m1 dex., 1,71 × 1,07 (PUI n¹); F, m1 dex., 1,71 × 1,08 (PUI n 4); G, m1 sen.,

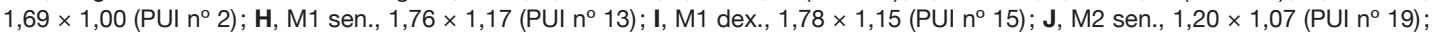
K, M2 sen., 1,27 × 1,13 (PUI n $\left.{ }^{\circ} 20\right)$; L-N, Megacricetodon minor (Lartet, 1851); L, m2 sen., 1,18 $\times$ 0,97 (PUI $\left.n^{\circ} 26\right)$; M, M2 sen., 1,11 × 1,05 (PUI n 27); N, M2 sen., 1,07 × 0,97 (PUI n $\left.{ }^{\circ} 28\right)$; O, Democricetodon aff. freisingensis Fahlbusch, 1964, M2 dex. fragmentée, $L>1,59$ (PUI $\left.n^{\circ} 30\right)$. Échelle: $1 \mathrm{~mm}$.

\section{Discussion}

Par les dimensions et la morphologie, ces quelques molaires rappellent, d'une part, celles de $M$. aegercii de Sansan (Baudelot 1972), bien qu'ici la m1 présente un centrolophide plus long, et d'autre part, certaines molaires attribuées au Dryomys sp. $\mathrm{n}^{\circ} 2$ de Vieux-Collonges (Mein 1958).

Cette espèce n'est pas reconnue dans le gisement de Blanquatère 1.

\section{Agre du gisement}

Lassociation faunique de Blanquatère 3 diffère de celle de Blanquatère 1, d'une part par sa richesse moindre et, d'autre part, par la présence de Miodyromys cf. aegercii et du genre Cricetodon, qui confèrent au gisement un âge un peu plus récent que Blanquatère 1. Les deux espèces $C$. meini et $C$. aureus bien représentées dans le gisement de Vieux-Collonges sont associées à deux espèces 
de Megacricetodon, $M$. collongensis et $M$. lappi (Mein, 1958) qui sont considérées comme plus évoluées que celles de Blanquatère 1 (Lazzari \& Aguilar 2007).

Blanquatère 3 occupe en conséquence une position intermédiaire entre le site de Blanquatère 1 et celui de Vieux-Collonges. Il représenterait actuellement, en France, le gisement le plus ancien ayant livré en Europe occidentale le genre Cricetodon, élément caractéristique de la biozone MN 5 (Mein 1999).

\section{LE GiSEMENT DE PUisSERGUIER (PUI)}

Commune de Puisserguier (Hérault).

Le Miocène de la région de Puisserguier (Hérault) a fait l'objet de nombreuses observations depuis le XIXe siècle par J. Miquel $(1896,1897,1899)$ et de nombreux fossiles y ont été découverts, mollusques marins et dents de requins, mollusques saumâtres ou lacustres, végétaux. Un bref historique a été effectué par Magné (1978). Les recherches de microfaunes marine et continentales datent des années 1970 (Aguilar \& Magné 1978; Magné 1978). Un nouveau site à micromammifères a été découvert (JPA) dans le talus en bordure du Chemin de Saint-Julien dont la coupe est à présent cachée par un mur de soutènement. On pouvait y observer, de bas en haut: 1$)$ calcaire blanc $(1,70 \mathrm{~m})$; 2) marno-calcaire verdâtre $(0,20 \mathrm{~m}) ; 3)$ marnes noires à gastéropodes et rongeurs $(0,15$ à $0,20 \mathrm{~m}) ; 4)$ marnes verdâtres $(1,10 \mathrm{~m}) ; 5)$ sol. Cette coupe ressemble à celle dite «du Four à Chaux» décrite par Magné (1978) qui est située $700 \mathrm{~m}$ environ au SO et sensiblement à la même altitude $(74 \mathrm{~m})$. Ces deux coupes sont en revanche altitudinalement plus basse que celle du Mourel de l'Oreille (103 m) où se situe le gisement à micromammifères de La Grenatière (Aguilar \& Magné 1977; Magné 1978; Aguilar 1980). Près de $300 \mathrm{~kg}$ de sédiments prélevés avec l'aide de Frank Sénégas et Claude Requirand ont donné par le lavage-tamisage une microfaune modeste représentée principalement par des rongeurs auxquels sont associés une dent d'insectivore (Galerix sp.), quelques fragments de dents de lagomorphes, d'artiodactyles, ainsi que des plaques dermiques et des dents de lézard. La faune de rongeurs se présente comme suit.

\section{Cricetodon cf. albanensis}

Mein \& Freudenthal, 1971

(Fig. 2D)

Matériel ET Dimensions. - Partie antérieure d'une m1 (PUI no 29).

\section{Description}

L'antéroconide est relié à la fois au métaconide par le métalophulide en position labiale et au protoconide, par l'antérolophulide. Il existe un antérolophide labial qui délimite un protosinuside assez marqué et on aperçoit le départ d'une crête labiale à l'arrière du protoconide. La largeur mesurée entre la base du métaconide et celle du protoconide est de $1,73 \mathrm{~mm}$.

\section{Discussion}

La largeur ici mesurée est bien supérieure à celle observée chez Cricetodon meini Freudenthal, 1963 de Vieux-Collonges (Mein \& Freudenthal 1971b) ainsi que chez Hipanomys castelnovi Aguilar, Calvet \& Michaux, 1994 (Aguilar et al. 1994) et Hispanomys bijugatus Mein \& Freudenthal, 1971 (Mein \& Freudenthal 1971a). C'est avec Cricetodon albanensis de La Grive M, présent dans les collections de Montpellier que cette partie antérieure de $\mathrm{m} 1$ présente le plus d'affinités. Cricetodon albanensis est également signalé à La Grive L7 (Mein \& Freudenthal 1971a; Mein \& Ginsburg 2002).

\section{Democricetodon aff. freisingensis Fahlbusch, 1964} (Fig. 2O)

MatéRIEL ET Dimensions. - 1 M2 fragmentée dont la longueur minimale est de 1,59 mm (PUI no 30).

\section{Description}

Cette M2 est caractérisée par un mésolophe très développé qui atteint le bord labial et développe un petit mésostyle. Le métalophule est postérieur, mais on note une ébauche de branche antérieure. Le protolophule paraît double.

\section{Discussion}

Cette morphologie est connue chez Democricetodon gaillardi de Sansan (Baudelot 1972) avec lequel nous 
avons pu comparer notre dent, mais également chez D. freisingensis signalé au CD 24, à La Grive L3 et L5 (Mein \& Ginsburg 2002). Democricetodon freisingensis présente des dimensions supérieures à celles de D. gaillardi (mesures in Maridet 2002). La dent fragmentée de Puisserguier montre une dimension minimale qui est légèrement supérieure au domaine de variation de D. gaillardi (Baudelot 1972) et qui paraît plus conforme aux mesures observées chez D. freisingensis.

Megacricetodon aff. fournasi Aguilar, 1995

(Fig. 2E-K; Tableau 3)

MATÉRIEL ET DIMENSIONS. - Dents isolées (PUI no 1 à 25). Dimensions: voir Tableau 3.

\section{Description}

Molaires inférieures. Sur m1, l'antéroconide est rond (2) ou légèrement divisé en sa partie apicale (2), une seule molaire montre un mésolophide très court et une autre molaire montre un petit éperon lingual qui rejoint la partie postérieure de l'antéroconide. Sur m2, l'antérolophide labial est bien développé et le mésolophide est absent (1), très court (3) ou de longueur moyenne (1) et rejoint la base du protoconide.

Molaires supérieures. Sur M1, l'antérocône est assez profondément fissuré, bien dédoublé avec deux cuspides sensiblement de même volume, le mésolophe de longueur moyenne relié à l'éperon postérieur du paracône (1). Cet éperon est absent sur une dent. Les liaisons sont rétroverses et il y a un petit postérosinus. Sur M2, le protolophule est simple et en position antérieure, le métacône est isolé sur une dent alors que sur l'autre, il est relié à l'hypocône par le métalophule en position antérieure.

\section{Discussion}

C'est l'espèce dominante dans le gisement (28 dents déterminables dont 25 mesurables), elle représente la presque totalité de la faune.

Ces molaires présentent des caractères morphologiques moins évolués que ceux de $M$. gregarius (Schaub,
TABleau 3. - Mensurations (en $\mathrm{mm}$ ) des molaires de Megacricetodon aff. fournasi Aguilar, 1995 du gisement de Blanquatère 3 (sud de la France). N, nombre de dents dont la longueur/largeur est mesurable.

\begin{tabular}{lcccccccc}
\hline & & \multicolumn{3}{c}{ Longueur } & & \multicolumn{3}{c}{ Largeur } \\
\cline { 6 - 7 } & $\mathbf{N}$ & min & moy & max & & min & moy & max \\
\hline m1 & $4 / 6$ & 1,61 & 1,68 & 1,71 & & 0,98 & 1,03 & 1,07 \\
m2 & $5 / 4$ & 1,27 & 1,29 & 1,34 & & 1,06 & 1,10 & 1,15 \\
m3 & 1 & & 1,29 & & & & \\
M1 & $2 / 4$ & 1,76 & 1,77 & 1,78 & & 1,14 & 1,17 & 1,20 \\
M2 & 2 & 1,20 & & 1,27 & & 1,07 & & 1,13 \\
M3 & 5 & 0,83 & 0,90 & 0,93 & & 0,89 & 0,93 & 0,96 \\
\hline
\end{tabular}

1944) du gisement voisin et stratigraphiquement plus récent de La Grenatière, avec notamment un antéroconide de $\mathrm{m} 1$ peu ou pas divisé.

La présence d'un antéroconide légèrement divisé sur deux des quatre $\mathrm{m} 1$ complètes et d'un antérocône assez profondément fissuré, permet de rapprocher morphologiquement cette petite population de celle de M. fournasi Aguilar, 1995 de Lo Fournas 2 qui présente de tels caractères alors que chez le stade moins évolué M. lemartineli Aguilar, 1995, l'antéroconide n'est jamais aussi nettement fissuré (Aguilar 1995).

En ce qui concerne les dimensions elles entrent dans les domaines de variation des espèces $M$. lemartineli et $M$. fournasi.

En conclusion, par la morphologie proche de $M$. fournasi et les dimensions intermédiaires entre $M$. fournasi et $M$. lemartineli, nous définirons cette petite population comme $M$. aff. fournasi.

Megacricetodon minor (Lartet, 1851)

(Fig. 2L-N)

MATÉRIEL ET Dimensions. $-1 \mathrm{~m} 2(1,18 \times 0,97)$ (PUI no 26); 2 M2 $(1,11 \times 1,05 ; 1,07 \times 0,97)($ PUI $\left.\mathrm{n}^{\circ} 27,28\right)$.

\section{Description}

Ces molaires présentent des dimensions inférieures à celles de $M$. aff. fournasi et sont comparables à celles de $M$. minor de La Grive M, présentes dans les collections de l'Université Montpellier 2. Sur m2 le mésolophide est plus long que chez $M$. aff. fournasi, il atteint le bord lingual; sur M2 le mésolophe est de longueur moyenne, le protolophule est double 
sur une dent et en position médiane sur l'autre, le métalophule étant antérieur. Le sinuside de la $\mathrm{m} 2$ et le sinus des M2 sont fermés par une crête.

\section{ÂGE DU GISEMENT}

L'association Cricetodon cf. albanensis, Democricetodon aff. freisingensis, Megacricetodon aff. fournasi et Megacricetodon minor, indique sans conteste le Miocène moyen pour la faune de Puisserguier. Megacricetodon aff. fournasi permet de plus de lui attribuer un âge sensiblement voisin de celui de Lo Fournas 2, et plus ancien que celui de La Grenatière.

\section{BIOCHRONOLOGIE ET CORRÉLATION (FIG. 3)}

L'association Cricetodon cf. albanensis, Democricetodon aff. freisingensis, Megacricetodon aff. fournasi et Megacricetodon minor est nouvelle pour le Languedoc-Roussillon qui a pourtant livré à ce jour un nombre considérable de sites (Aguilar et al. 1999b). En effet, le genre Cricetodon n'était connu à ce jour qu'à Luc-sur-Orbieu (localité qui n'est pas issue d'un remplissage karstique); ce genre est à nouveau signalé, à Blanquatère 3. À Luc-sur-Orbieu, c'est l'espèce C. sansaniensis Lartet, 1851 qui est représentée alors qu'à Blanquatère 3 ce sont $C$. meini et C. aureus (présent travail). Si le genre Democricetodon est abondamment représenté dans les faunes du Miocène moyen, l'espèce $D$. aff. freisingensis n'avait pas été signalée jusqu'ici. Megacricetodon aff. fournasi n'est connu par ailleurs que de Lo Fournas 2 et Cases-de-Pènes, sur le Plateau de Baixas.

En revanche, $C$. cf. albanensis et $D$. aff. freisingensis sont connus dans les gisements de la région lyonnaise, à La Grive $\mathrm{M}$ et L7 pour la première espèce, au CD 24 et à La Grive L3 et L5 pour la seconde espèce (Mein \& Ginsburg 2002). À l'inverse, la lignée Megacricetodon «collongensis»-roussillonensis, reconnue en Languedoc-Roussillon (Aguilar 1995) ne semble pas représentée dans la région lyonnaise; en effet, à La Grive M, L7 et CD 24, le genre Megacricetodon est représenté par l'espèce $M$. gregarius alors qu'à La Grive L3 et L5, c'est l'espèce $M$. aff. ibericus (Schaub, 1944).
Megacricetodon gregarius, qui appartient à une lignée différente de celle de $M$. "collongensis»-rousssillonensis (Aguilar 1995), est cependant connu en Languedoc et plus particulièrement dans le secteur de Puisserguier, dans le gisement de La Grenatière (Aguilar \& Magné 1978; Aguilar 1980) situé à une altitude supérieure (approximativement $30 \mathrm{~m}$ ) et à $2 \mathrm{~km}$ environ du nouveau gisement. La population de La Grenatière paraît plus évoluée que celle de La Grive M (Aguilar 1980).

L'espèce $M$. fournasi est plus évoluée que celle de $M$. gersii reconnue à Sansan et dans plusieurs localités du Roussillon.

Le gisement de Puisserguier est plus ancien par sa faune que celui de La Grenatière, ce que confirme la stratigraphie locale. Il est plus jeune que celui de Sansan et également équivalent, par sa faune, de l'un des niveaux de La Grive sans plus de précision car l'association, $C$. albanensis et $D$. aff. freisingensis, n'est signalé dans aucun des sites. Nous remarquerons que la position chronologique des sites de $\mathrm{La}$ Grive est controversée (La Grive M, L7, L3, L5 pour Mein \& Ginsburg [2002], La Grive L3, L5, L7, M pour Maridet [2002]).

\section{CORRÉLATIONS AVEC LE MARIN RÉGIONAL}

Le Miocène de l'Ouest biterrois suscite l'intérêt depuis la fin du XIXe siècle (voir historique in Magné 1978). Pour Magné, le Miocène marin du Languedoc débute par des dépôts saumâtres à laguno-lacustres (Aquitanien), se poursuit par des dépôts franchement marins, du Burdigalien au Langhien, compris entre la zone N5 et la zone N8 (niveaux à Praeorbulina glomerosa (Blow, 1956)). Ces derniers ne représentent pas les niveaux les plus élevés, car les conditions de dépôts sont défavorables pour les foraminifères planctoniques. En revanche, ces niveaux plus élevés ont livré des nannofossiles des zones NN5 et NN5/6 (C. Muller in litt.) dans les coupes de Montady et de la carrière de Sériège (Cruzy). Le niveau le plus récent reconnu est représenté toutefois par la formation saumâtre de La Grenatière qui est attribuée au Serravallien (Aguilar \& Magné 1977).

Ainsi, dans le Miocène de Capestang, l'association P. glomerosa, G. cf. sicanus de Stephani, 1950 caractérisant la biozone N8 (Berggren et al. 1995) 


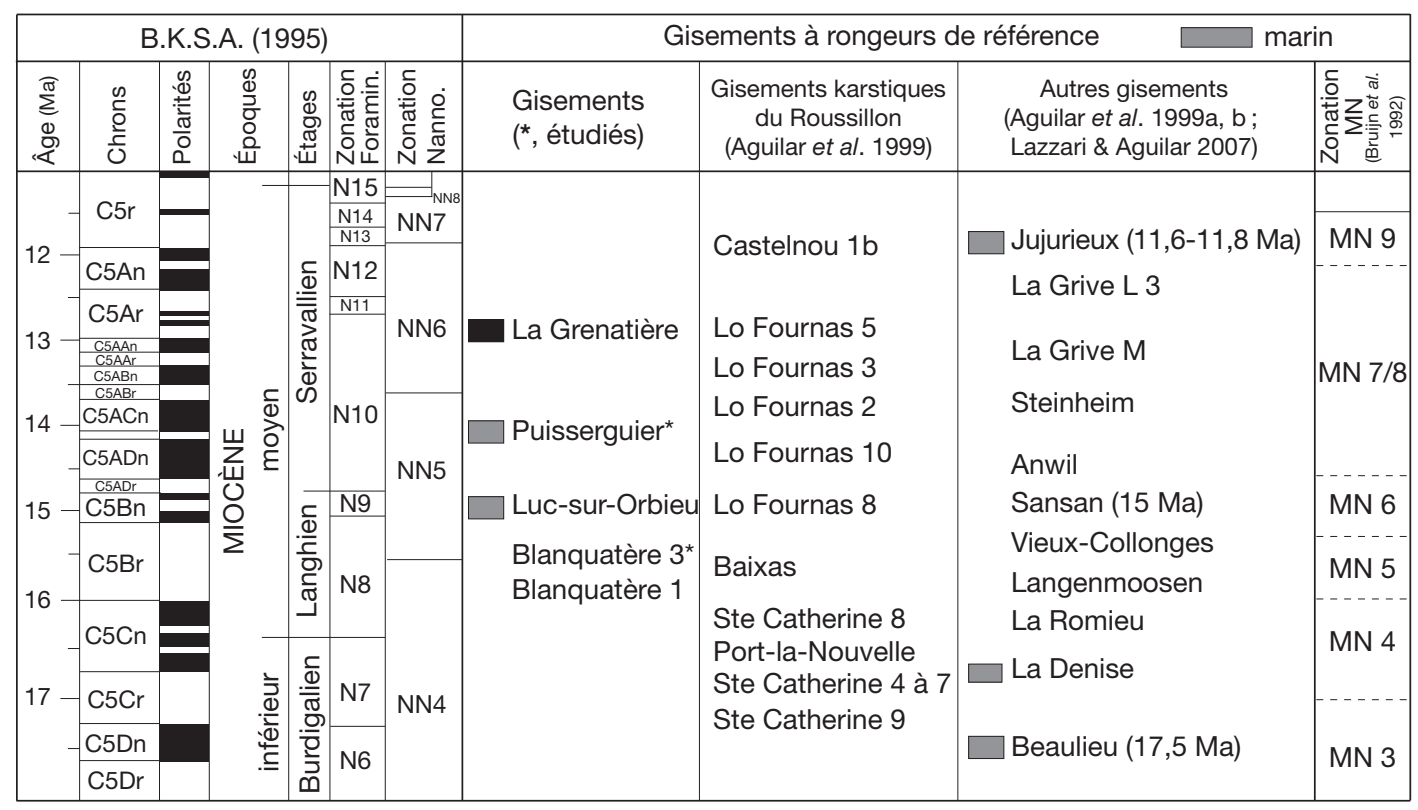

FIG. 3. - Biochronologie des gisements du Miocène moyen en Languedoc-Roussillon et corrélations avec les sites de la région lyonnaise. B.K.S.A., Berggren, Kent, Swisher, Aubry.

est reconnue à $15 \mathrm{~m}$ du sommet de la coupe située à 2,5 km environ des coupes de Puisserguier (Magné 1978). Bien qu'il n'y ait pas d'affleurement continu entre ces deux coupes les formations représentées relèvent du même ensemble (voir Anonyme 1982), la partie sommitale étant représentée par une formation laguno-lacustre datée par les rongeurs seulement à La Grenatière.

Le sondage en mer de Tramontane 1, situé à $30 \mathrm{~km}$ à l'est de Leucate et à $60 \mathrm{~km}$ environ de Capestang et de Puisserguier (Cravatte et al. 1974) indique que le Miocène moyen y est représenté par des niveaux à Praeorbulina Olssen, 1964 et à Orbulina universa d'Orbigny, 1839 qui définissent les biozones N8 et N9, les dépôts devenant ensuite littoraux puis saumâtres. C'est donc une même succession qui s'observe à terre, dans la région de Puisserguier et de Capestang. Les deux séquences du Miocène moyen qui se terminent par des faciès régressifs, peuvent être rapportées à titre provisoire à la séquence TB 2.5 (Haq et al. 1987; Hardenbol et al. 1998). En conséquence, le gisement de La Grenatière devrait se voir attribuer un âge, au plus jeune de $13 \mathrm{Ma}$, celui de Puisserguier un âge de $14 \mathrm{Ma}$ environ. Ces observations renforcent encore la proposition d'un âge de 15 Ma donnée pour le gisement de Sansan sur la base de la magnétostratigraphie (Sen 1996, 1997). Un tel âge est également celui que nous avons donné pour le gisement marin de Luc-sur-Orbieu sur la base de la faune de rongeur (Aguilar \& Magné 1978; Aguilar et al. 1994).

\section{CONCLUSION}

La découverte dans le sud de la France de deux nouvelles localités qui ont livré une faune de rongeurs, permet de compléter le registre fossilifere déjà bien documenté pour le Miocène moyen de cette zone. Le genre Cricetodon est reconnu une deuxième fois dans la région. Blanquatère 3 est plus ancien que le gisement de Luc-sur-Orbieu, Puisserguier est plus récent. La présence du genre Cricetodon permet de conforter les corrélations proposées jusqu'ici entre les gisements du sud de la France et ceux de la région lyonnaise. 
Le gisement de Blanquatère 3 représente, à ce jour, la localité la plus ancienne ayant livré le genre Cricetodon en France.

Le gisement de Puisserguier, interstratifié dans des niveaux marins, est plus ancien que celui, géographiquement très proche de La Grenatière, plus récent que celui de Sansan et contemporain de l'un des sites de La Grive (La Grive M ?). Les gisements de Puisserguier et de la Grenatière que l'on peut situer dans le Miocène moyen marin du Languedoc permettent de proposer des âges numériques pour certains gisements à rongeurs: $13 \mathrm{Ma}$ pour La Grenatière, $14 \mathrm{Ma}$ pour Puisserguier et $15,5 \mathrm{Ma}$ pour Blanquatère 3. Ils appuient la proposition formulée par Sen $(1996,1997)$ sur l'âge de Sansan (15 Ma). Le Megacricetodon récolté à Puisserguier s'inscrit sans la modifier dans l'histoire du genre telle qu'elle avait été avancée par Aguilar (1995) pour le sud de la France.

\section{Remerciements}

Nous remercions les deux rapporteurs anonymes pour leurs remarques constructives.

Publication ISE-M-2010 - 004 (UMR 5554, CNRS).

\section{RÉFÉRENCES}

Aguilar J.-P. 1980. — Rongeurs du Miocène inférieur et moyen en Languedoc. Leur apport pour les corrélations marin-continental et la stratigraphie. Palaeovertebrata 9 (6): 155-203.

Aguilar J.-P. 1982. - Biozonation du Miocène d'Europe occidentale à l'aide des rongeurs et corrélations avec l'échelle stratigraphique marine. Comptes rendus de l'Académie des Sciences 294, série II: 49-54.

Aguilar J.-P. 1995. - Évolution de la lignée Megacricetodon collongensis-Megacricetodon roussillonensis (Cricetidae, Rodentia, Mammalia) dans le sud de la France. Palaeovertebrata 24 (1): 1-45.

Aguilar J.-P. 1999. — Les rongeurs de deux sites karstiques du Miocène moyen des Pyrénées-Orientales (Sud de la France) avec une nouvelle espèce de Pseudofalhbuschia (Rodentia, Mammalia). Eclogae Geologicae Helvetiae 92: 475-481.

Aguilar J.-P. 2002. - Les sciuridés des gisements karstiques du Miocène inférieur à moyen du sud de la France: nouvelles espèces, phylogénie, paléoenvironnement. Geobios 35: 375-394.
Aguilar J.-P. \& Magné J. 1977. — Présence de rongeurs dans le Miocène marin et laguno-lacustre de La Grenatière (commune de Puisserguier, Hérault). Compte rendu sommaire de la Société géologique de France 1: 27-28.

Aguilar J.-P. \& Magné J. 1978. — Nouveaux gisements à rongeurs dans des formations marines $\mathrm{du}$ Languedoc méditerranéen. Bulletin de la Société géologique de France 20 (6): 803-805.

Aguilar J.-P. \& Michaux J. 1987. — Essai d'estimation du pouvoir séparateur de la méthode biostratigraphique des lignées évolutives chez les rongeurs néogènes. Bulletin de la Société géologique de France (8), III, 6: 1113-1124.

AgUiLAR J.-P. \& LAZZARI V. 2006. — Nouvelles espèces de gliridés du gisement karstique de Blanquatère 1 (Miocène moyen, sud de la France). Geodiversitas 28 (2): 277-295.

Aguilar J.-P., Calvet M. \& Michaux J. 1994. Les rongeurs de Castelnou 6 (Pyrénées-Orientales, France) et les corrélations entre faunes ibériques et françaises au Miocène moyen. Neues Jahrbuch für Geologie und Paleontologie, Monatshefte 192 (1): 109-131.

Aguilar J.-P., Clauzon G. \& Michaux J. 1999a. Nouveaux cricétidés (Rodentia, Mammalia) dans le Miocène moyen de la région de Digne (Alpes de Haute Provence). Systématique, Biochronologie, Corrélations. Palaeontographica A 253: 1-28.

Aguilar J.-P., Escarguel G. \& Michaux J. 1999b. A succession of Miocene rodent assemblages from fissure fillings in southern France: palaeoenvironmental interpretation and comparison with Spain. Palaeogeography, Palaeoclimatology, Palaeoecology 145: 215-230.

Aguilar J.-P., Michaux J., Aunay B., Calvet M. \& LAZZARI V. 2010. - Compléments à l'étude des Rongeurs (Cricetidae, Eomyidae, Sciuridae) du gisement karstique de Blanquatère 1 (Miocène moyen, Sud de la France). Geodiversitas 32 (3): 515-533.

ANONYME 1982. - Carte géologique de Béziers au $1 / 50000$. BRGM no 2545.

BAUDELOT S. 1972. - Étude des Chiroptères, Insectivores et Rongeurs du Miocène de Sansan (Gers). Thèse, Université P. Sabatier, Toulouse, 364 p.

Berggren W. A., Kent D. V., Swisher C. C. \& Aubry M.-P. 1995. - A revised Cenozoic geochronology and chronostratigraphy, in BERGGREN W. A., KENT D. V. \& HARDENBOL J. (eds), Geochronology, time scales and global stratigraphic correlations: a unified temporal framework for an historical geology. Society of Economic Paleontologists and Mineralogists, Special Volume no. 54: $126 \mathrm{p}$.

Cravatte J., Dufaure P., Prim M. \& Rouaix S. 1974. - Les sondages du Golfe du Lion: strati- 
graphie sédimentologique. Notes et Mémoires de la Compagnie française de Pétroles 11: 209-274.

FaHLBUSCH V. 1964. - Die Cricetiden der oberen Süsswassermolasse Bayerns. Bayerische Akademie der Wissenschaften, Mathematisch-naturwissenschaftliche Klasse, München, Abhandlungen N.F. 118: 136 p.

Freudenthal M. \& DaAms R. 1988. - Cricetidae (Rodentia) from the type-Aragonian; the genera Democricetodon, Fahlbuschia, Pseudofablbuschia nov. gen., and Renzimys, in Freudenthal M. (ed.), Biostratigraphy and paleoecology of the Neogene micromammalian faunas from the Calatayud-Teruel Basin (Spain). Scripta Geologica Special Issue: 133-252.

Haq B. U., Hardenbol J. \& Vail P. R. 1987. Chronology of fluctuating sea levels since the Triassic. Science 235: 1156-1167.

HaRdenbol J., Thierry J., Farley M. B., JaCQUin T., de Graciansky P.-C. \& Vail P. R. 1998. - Mesozoic and Cenozoic sequence chronostratigraphic chart, in DE GRACIANSKY P.-C., HARDENBOL J., JACQUIN T. \& VAIL P. R. (eds), Mesozoic and Cenozoic sequence chronostratigraphic framework of European basins. Society of Economic Paleontologists and Mineralogists, Special Publication 60.

LaZZari V. \& Aguilar J.-P. 2007. — Les Megacricetodon du gisement karstique Miocène moyen de Blanquatère 1 (Pyrénées-Orientales, sud de la France): nouvelles espèces, implication phylogénique. Geobios 40: 91-111.

LazZari V., Michaux J. \& Aguilar J.-P. 2007. — First occurrence in Europe of Myocricetodontinae (Rodentia, Gerbillidae) during the Lower Middle Miocene in the karstic locality of Blanquatère 1 (Southern France): implications. Journal of Vertebrate Paleontology 27 (4): 1062-1065.

MAGNÉ J. 1978. - Études microstratigraphiques sur le Néogène de la Méditerranée nord-occidentale: Partie 2 - Le Néogène du Languedoc méditerranéen. Thèse, Université P. Sabatier, Toulouse, France, $435 \mathrm{p}$.

MARIDET O. 2002 - Révision du genre Democricetodon (Mammalia, Rodentia, Cricetinae) et dynamique des faunes de rongeurs du Néogène d'Europe occidentale : évolution, paléobiodiversité et paléobiogéographie. Thèse, Université C. Bernard, Lyon I, France, $252 \mathrm{p}$.
MEIN P. 1958 - Les mammifères de la faune sidérolithique de Vieux-Collonges. Nouvelles Archives du Muséum d'Histoire Naturelle de Lyon 5: 1-122.

MeIN P. 1999. - European Miocene Mammal Biochronology, in RÖSSNER G., HEISSIG K. (eds), The Miocene Land Mammals of Europe. Verlag Dr Friedrich Pfeil, München: 25-38.

Mein P. \& FreudentHAL M. 1971a. — Une nouvelle classification des Cricetidae (Mammalia, Rodentia) du Tertiaire d'Europe. Scripta Geologica 2: 1-37.

Mein P. \& Freudenthal M. 1971b. - Les Cricetidae (Mammalia, Rodentia) du Néogène moyen du Vieux-Collonges. Partie $1:$ Le genre Cricetodon Lartet, 1851. Scripta Geologica 5: 1-51.

Mein P. \& GinsBURG L. 2002. - Sur l'âge relatif des différents dépôts karstiques miocènes de La Grive-Saint-Alban. Cabiers scientifiques - Muséum d'Histoire naturelle de Lyon (2): 7-47.

MiQuel J. 1896. - Notes sur la géologie des terrains secondaires et des terrains tertiaires du département de l'Hérault. Bulletin de la Société d'Etudes des Sciences naturelles de Béziers 19: 1-46.

MiQuel J. 1897. - Notes sur la géologie des terrains tertiaires du département de l'Hérault. Le Miocène dans le canton de Capestang et la vallée de Cruzy. Bulletin de la Société d'Études des Sciences naturelles de Béziers 20: 1-41.

MiQUEL J. 1899. — Notes sur la géologie des terrains secondaires et des terrains tertiaires du département de l'Hérault. Une journée d'exploration dans la commune de Puysserguier. Bulletin de la Société d'Études des Sciences naturelles de Béziers 22: 1-8.

SEN S. 1996. - Present State of Magnetostratigraphic Studies in the Continental Neogene of Europe and Anatolia, in BERNOR R. L., FAHLBUSCH V. \& MitTmann H.-W. (eds), The Evolution of Western Eurasian Neogène Mammal Faunas. Columbia University Press, New York: 56-63.

SEN S. 1997. - Magnetostratigraphic calibration of the European Neogene mammal chronology. Palaeogeography, Palaeoclimatology, Palaeoecology 133: 181-204.

WU W. 1982. - Die Cricetiden (Mammalia, Rodentia) aus der Oberen Süsswasser-Molasse von Puttenhausen (Niederbayern). Mitteilungen der Bayerischen Staatssamlung für Paläontologie und Historische Geologie 30: 65-105. 